\title{
Is visual short-term memory object based? Rejection of the "strong-object" hypothesis
}

\author{
INGRID R. OLSON \\ Yale University, New Haven, Connecticut \\ and \\ YUHONG JIANG \\ Massachusetts Institute of Technology, Cambridge, Massachusetts
}

\begin{abstract}
Is the capacity of visual short-term memory (VSTM) limited by the number of objects or by the number of features? VSTM for objects with either one feature or two color features was tested. Results show that capacity was limited primarily by the number of colors to be memorized, not by the number of objects. This result held up with variations in color saturation, blocked or mixed conditions, duration of memory image, and absence or presence of verbal load. However, conjoining features into objects improved VSTM capacity when size-orientation and color-orientation conjunctions were tested. Nevertheless, the number of features still mattered. When feature heterogeneity was controlled, VSTM for conjoined objects was worse than VSTM for objects made of single features. Our results support a weak-object hypothesis of VSTM capacity that suggests that VSTM is limited by both the number of objects and the feature composition of those objects.
\end{abstract}

Visual information can be held for short amounts of time in a store termed visual short-term memory (VSTM). It is distinguished from iconic memory (Phillips, 1974; Sperling, 1960), and separate from the verbal short-term store (Baddeley, 1986; Logie, 1995). Yet, just like verbal short-term memory (STM), VSTM has severe capacity limits (Cowan, 2001; Lee \& Chun, 2001; Luck \& Vogel, 1997; Pashler, 1988; Phillips, 1974; Simons, 1996). The capacity of VSTM for simple visual features is around four units (Luck \& Vogel, 1997). The capacity for spatial locations is slightly larger but still limited, at around six locations (Simons, 1996). To use such limited capacity efficiently, the visual system has developed strategies or rules to represent information in VSTM (Jiang, Olson, \& Chun, 2000).

Recent experiments by Luck and Vogel (1997), reported more fully by Vogel, Woodman, and Luck (2001), revealed an important strategy to increase the amount of information stored in VSTM. These researchers showed that multiple dimensions of a single object could be chunked together, via conjunction, as a single unit in VSTM, suggesting that, in VSTM, higher order units can be formed from smaller or simpler units (Miller, 1956). Luck and Vogel's

The authors thank Marvin Chun, Andrew Hollingsworth, Frank Keil, Martha Johnson, Steven Luck, and Mary Wheeler for helpful comments and discussions. Correspondence should be addressed to I. R. Olson, Department of Diagnostic Radiology, Yale School of Medicine, 333 Cedar St., Fitkin Bldg., RM F14, New Haven, CT 06510 (e-mail: iolson@ boreas.med.yale.edu), or to Y. Jiang, Department of Brain and Cognitive Sciences, MIT, NE20-443, 77 Massachusetts Ave., Cambridge, MA 02139 (e-mail: yuhong@mit.edu). results showed that memory for the conjunction of multiple features, such as color and orientation, was as good as memory for each simple feature. For example, observers were able to hold approximately four colors or four oriented lines in VSTM but not eight of each type. However, all eight features could be remembered when four colors and four orientations were conjoined to form four colored lines. Luck and Vogel proposed that VSTM was based on object representations. They suggest that VSTM has capacity limits defined by the number of objects to be memorized, not the number of features. Multiple dimensions of the same object can be stored in VSTM at no additional cost.

The object-based memory mechanism fits well with what we know about STM and visual attention. Studies on verbal STM have obtained a great deal of evidence for chunking of digits, letters, and words (e.g., Bower \& Winzenz, 1969; Ericsson, Chase, \& Faloon, 1980; Miller, 1956; Simon, 1974). The same general chunking principle applies to VSTM as well: VSTM for spatial locations, color, and shape relies on configural representation (Jiang et al., 2000). In addition, studies on visual attention suggest that conjoining different features into a single object reduces the number of locations to be encoded, thereby allowing several features to be attended without interference (Duncan, 1984; Egly, Driver, \& Rafal, 1994; Vecera \& Farah, 1994). Objecthood thus appears to have special status in visual representations (see also Irwin \& Andrews, 1996, for object-based representation in transsaccadic memory).

An alternative to the object-based theory of VSTM is the multiple-resources theory, which assumes that there are separate pools of resources for remembering different 
feature types, such as orientation, size, and color. Observers can remember four colored oriented lines because they use two different pools of memory, one for color and the other for orientation. There is no need to recruit the objectbased memory mechanism. The notion of limited resources for encoding each feature dimension is supported in studies of visual attention. Wolfe et al. (1990) compared search efficiency for targets defined by single features and feature conjunctions. They found that visual search for conjunction of two colors was less efficient than conjunction of color and orientation. They propose that visual search operates along channels for feature dimensions, such as color and orientation. The same principle may hold for VSTM.

In the present study, we attempted to replicate Luck and Vogel's (1997) and Vogel et al.'s (2001) results for memory conjunctions. We asked the question: Is there any cost in storing multiple features as compared with storing a single feature of an object? Luck and Vogel's results imply that the number of features is irrelevant to VSTM and that only the number of objects matters. In contrast, studies of scene recognition show that visual memory is coarse in that details of a scene are typically lost in visual memory (Intraub, 1997), suggesting that the visual system may not have an unlimited capacity for storing features of an object.

To test whether multiple features of a single object can be stored in VSTM at no extra cost, we carried out four experiments, focusing on the conjunction of various features. We tested color-color conjunctions in Experiments 1 and 2, size-orientation conjunctions in Experiment 3, and color-orientation conjunctions in Experiment 4. These experiments were designed to test three competing hypotheses about VSTM. The first one was the strongobject hypothesis, according to which, VSTM only exerts capacity limits on the number of objects, not the number of features. Thus, the number of features memorized in VSTM should be twice as many for the conjunction of two features than for single features. The second hypothesis was the strong-feature hypothesis. Here, the number of features limits VSTM capacity. As such, the conjunction of features into objects provides no benefit. The same number of features should be remembered in VSTM regardless of whether features are separated or conjoined into objects. The multiple-resources theory falls under the umbrella of the strong-feature hypothesis. A third possibility is that VSTM may be limited in the number of features to be remembered but that feature conjunction can help alleviate this limitation through the grouping of features into higher order chunks. The efficiency of object chunking may depend on how features are conjoined and on how different the features are from one another. We call this hypothesis the weak-object hypothesis. It predicts superior performance in the conjunction condition relative to the feature condition, but the benefit may be less than doubling VSTM capacity for features. Our objective was to qualify the strong-object hypothesis in conditions that involved various types of feature conjunctions.

\section{EXPERIMENT 1 Color-Color Conjunction: A Failure to Replicate}

Strongest evidence for the strong-objecthypothesiscomes from the color-color conjunction experiment in Luck and Vogel's (1997) study. Observers were asked to remember the colors of objects. In one condition, each object was composed of one color. In another, each object was composed of two colors in a center-surround configuration. The memory display was briefly presented and followed by a probe display after a short retention interval.

The probe display was either identical to the memory display or different in the change of one color. Observers had to decide whether the colors at each location on the probe display were identical to the colors that had been in those positions on the memory display. The results showed that observers could remember four colors when each color was an object and could remember eight colors when the colors were conjoined to form four objects. This suggests that the unit of VSTM is the number of objects, not the number of features, supporting the strong-object hypothesis.

This result provides compelling evidence for the strongobject hypothesis. Compared with experiments that use conjoined features from different feature dimensions, results from color-color conjunction are less susceptible to alternative interpretations, such as that posed by the multipleresources theory. However, failures to replicate the benefit for color-color conjunctions have been noted (Wheeler \& Treisman, 1999; Xu \& Potter, 1999). Given its theoretical importance, we sought to replicate the effect of colorcolor conjunctions in VSTM.

In Experiment 1, we tested VSTM of colors. Two main types of color objects were tested: color-color conjunctions and color single-feature objects. The conjunction object was produced by two color squares in a centersurround configuration. The single-feature object was a unique color taken from either the inner or the outer color of the conjunction object. The strong-object hypothesis predicts that, if the number of objects is held constant, then VSTM should be the same for the conjunction object as for the single-feature object. Our main purpose was to find out whether such results are replicable.

VSTM of colors was tested in a probe task. Following the memory image and a brief retention interval, a single color probe was presented at one of the locations previously occupied by one of the memory objects. The observers had to decide whether the color of the probe was identical to the color of the memory object at that position.

Additionally, we examined how a color-color conjunction object was remembered. We presented two types of probes following a memory display of conjunction objects. One probe type consisted of two colors in a centersurround configuration. Another probe type was a singlefeature probe of either the inner or the outer color. On the basis of the encoding specificity hypothesis (Tulving \& Thomson, 1973), if the observers had encoded the two col- 
ors of the conjunction object in relation to each other, their memory should be impaired if one of the colors was absent in the probe. In contrast, if the observers had encoded the two colors independently of each other, their memory should be identical, regardless of whether the probe was a conjunction object or a single feature.

\section{Method}

Participants. Nine naive undergraduate students from Yale University participated in this experiment for course credit. Their ages ranged from 18 to 21 years. All had normal color vision and normal or corrected-to-normal visual acuity.

Materials. The stimuli appeared within an invisible $8 \times 8$ cell matrix that subtended $15^{\circ} \times 15^{\circ}$ of visual angle. The stimuli appeared on a uniform gray background. Two types of colored squares were used as stimuli in Experiments 1 and 2. The single-colored objects had only one color that was either an outer color frame $\left(1.56^{\circ} \times\right.$ $1.56^{\circ}$ in size; $0.40^{\circ}$ in the frame width), with a hollow center, or a small color square that was the same size as the inner hollow of the color frame $\left(0.76^{\circ} \times 0.76^{\circ}\right)$. A color-color conjunction object was composed of an outer color frame $\left(1.56^{\circ} \times 1.56^{\circ}\right.$ in size; $0.40^{\circ}$ in the frame width) and an inner color square $\left(0.76^{\circ} \times 0.76^{\circ}\right)$.

Eight colors were used for the stimuli. They were produced by permutation of the presence or absence of red, blue, and green phosphors (i.e., RGB colors). The RGB colors were on a 0-255 scale, in which 0 represented $0 \%$ intensity and 255 represented $100 \%$ intensity. The colors were as follows: black (RGB:0,0,0), white $(255,255,255)$, red $(255,0,0)$, pink $(255,0,255)$, yellow $(255,255,0)$, green $(0,255,0)$, blue $(0,0,255)$, and cyan $(0,255,255)$. All colors were highly distinctive. The colors used on the same display were allowed to repeat, with the constraint that the inner and outer colors of the same object were different and that the inner colors of different objects were different from one another and the outer colors were also different from one another.

Procedure. VSTM of colors was tested in a probe change detection paradigm. Each trial was composed of a memory display and a probe display separated by a brief blank interval. The memory display contained three or six colored objects presented at random locations. The probe display contained one colored object. The observers' task was to detect whether the color of the probe object was the same as the color located at that position on the memory display.

There were two types of memory objects: single-color objects ( $1 / 3$ of the trials) and color-color conjunction objects ( $2 / 3$ of the trials). A single-color object was shaped either as an outer color frame or as an inner color square. A color-color conjunction object was composed of an outer color frame enclosing an inner color square. Luck and Vogel (1997) also used a center-surround configuration in their color-color conjunction experiment. The number of objects on the memory display was either three or six. Objects on the same memory display were always of the same type (simple or conjunction). Thus, holding the number of objects constant, the color-color conjunction condition contained twice as many colors as the simple feature condition.

When the memory display contained simple objects, its corresponding probe display always contained a simple object of the same type (outer frame or inner square). We call this the feature-feature condition. On the color-color conjunction memory displays, the corresponding probe display took two forms. On half of the conjunction trials, the probe item was a conjunction object. This was the conjunction-conjunction condition. Only one of the colors could change (either the outer frame or the inner square). In the other half of the conjunction trials, the probe item was a simple object (either an outer frame or an inner square). This was the conjunction-feature condition. A sample of the three conditions is illustrated in Figure 1.

The observers initiated each trial by a barpress. A white fixation point $\left(0.2^{\circ} \times 0.2^{\circ}\right)$ was presented for $400 \mathrm{msec}$, followed by the memory display of $400 \mathrm{msec}$. After a blank retention interval of $907 \mathrm{msec}$, the probe display was presented until a response key was pressed. The observers were instructed to memorize all the colors presented on the memory display and to detect whether the color(s) of the probe was (were) the same as the memory color(s) at that location. Sound feedback concerning the accuracy of response was provided after each trial.

Each observer completed 12 practice trials and 288 experimental trials (i.e., 3 [conditions] $\times 2$ [probe change absent vs. present] $\times 2$ [inner square or outer frame as the possible change feature] $\times 24$ cases). Conditions were interleaved, and trials were presented in a random order.

Equipment. All observers were tested individually in a room with normal interior lighting. All experiments were carried out on a Macintosh computer with a 17-in. screen, using MacProbe software (Hunt, 1994). The unrestricted viewing distance was approximately $57 \mathrm{~cm}$, at which the viewing distance of $1 \mathrm{~cm}$ corresponded to $1^{\circ}$ of visual angle.

\section{Results}

In the following analysis, we used percentage correct of the change detection performance as the dependent measure. Past researchers calculated the capacity of VSTM using various procedures. One estimate of VSTM capacity is based on a function relating percentage correct to memory set size (Pashler, 1988; Vogel et al., 2001). Another estimate is based on a nonparametric measure of memory sensitivity (Xu \& Potter, 1999; see discussion of $A^{\prime}$ in Macmillan \& Creelman, 1991). Because we were interested in how objecthood affected memory accuracy rather than exactly how many units could be stored in VSTM, we did not think it was necessary to measure the exact capacity of VSTM. Instead, we performed inferential statistical tests on percentage correct across conjunction and feature conditions, holding the number of units comparable in the comparison.

Assessment of strong-object hypothesis. Mean accuracy as a function of the number of to-be-remembered objects and conditions is plotted in Figure 2. A repeated measures analysis of variance (ANOVA) tested the effects of condition and the number of objects. The main effect of condition was significant $[F(2,16)=67.22, p<.001]$, as was the main effect of set size, with better performance at smaller set sizes $[F(1,8)=33.38, p<.001]$, but the interaction between condition and set size was not significant $[F(2,16)=2.29, p>.10]$. Planned comparisons between the three conditions showed that, when holding the number of objects constant, performance was better in the feature-feature condition than in both the conjunctionconjunction condition $[F(1,8)=38.00, p<.001]$ and the conjunction-feature condition $[F(1,8)=129.88, p<$ $.001]$. Thus, when holding the number of objects constant, VSTM was better for objects of simple colors than for objects of color-color conjunctions. In addition, performance in the conjunction-conjunction condition was significantly better than that in the conjunction-feature condition $[F(1,8)=29.34, p<.001]$. This suggests that the observers had encoded the two colors of the conjunction object in relation to each other. Such relational processing, however, was not sufficient to double the capac- 

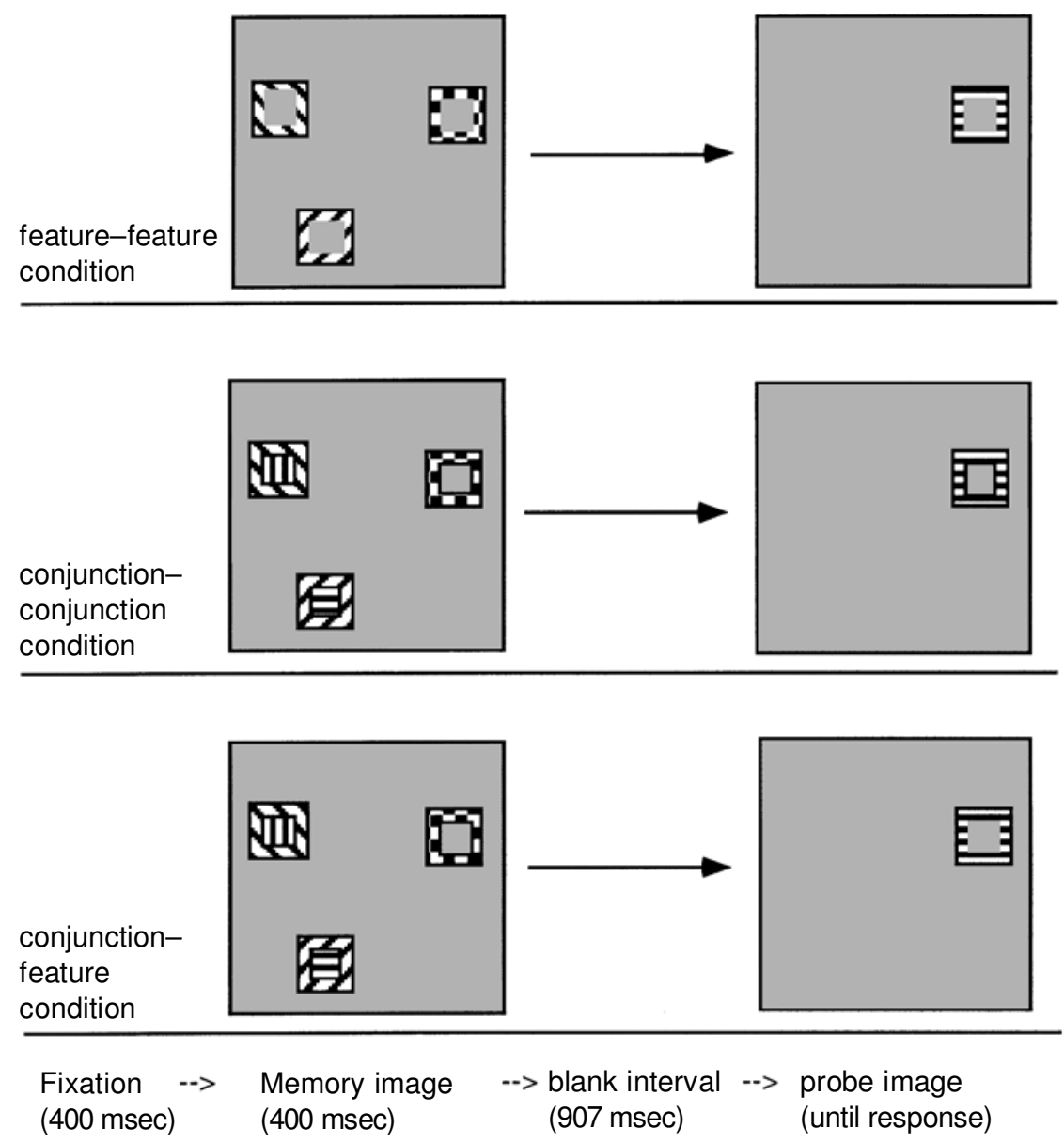

Figure 1. Sample displays used in Experiment 1. Different textures on different squares represent different colors used in the experiment. In the actual experiment, the texture of every object was smooth; objects and features differed only in color.

ity of VSTM for color-color conjunction objects. Holding the number of objects constant, VSTM performance was poorer when two colors rather than one were to be memorized, indicating that the number of features matters, invalidating the strong-object hypothesis.

Assessment of the strong-feature hypothesis. The analysis above did not tell us whether there was any benefit of color-color conjunction in terms of the number of features remembered. This was determined by holding the number of features constant across the three conditions. In the feature-feature condition, the observers tried to remember three or six features. In the two conjunction conditions, the observers tried to remember six or 12 features. To assess the effect of color conjunction, we tested the difference between conditions when six features were to be remembered. There was a main effect of condition $[F(2,16)=8.03, p<.004]$. Specifically, performance in the conjunction-feature condition was significantly worse than that in the other two conditions $[t \mathrm{~s}(8)>2.98, p \mathrm{~s}<$ .018], again supporting the encoding specificity hypothesis that the two colors in the conjunction object were processed in relation to each other. Importantly, perfor- mance in the feature-feature condition did not differ significantly from that in the conjunction-conjunction condition $[t(8)<1]$. There was no improvement of VSTM when two colors were presented in a center-surround configuration rather than when they were presented separately as single features. This occurred even when evidence suggests that the two colors in the conjunction object were processed in relation to each other.

\section{Discussion}

In a color-color conjunction VSTM experiment, we failed to replicate the object-based VSTM representation as observed by Luck and Vogel (1997). Holding the number of objects constant, VSTM was clearly better in the simple-feature condition than in the color-color conjunction condition. Storing two colors of one object was much more difficult than storing one color feature. This is inconsistent with the hypothesis that VSTM has a capacity that is equal for conjunctions as it is for single features.

We not only failed to find doubled capacity for conjunction of two features but also failed to find any benefit of putting two colors in a center-surround conjunction. 


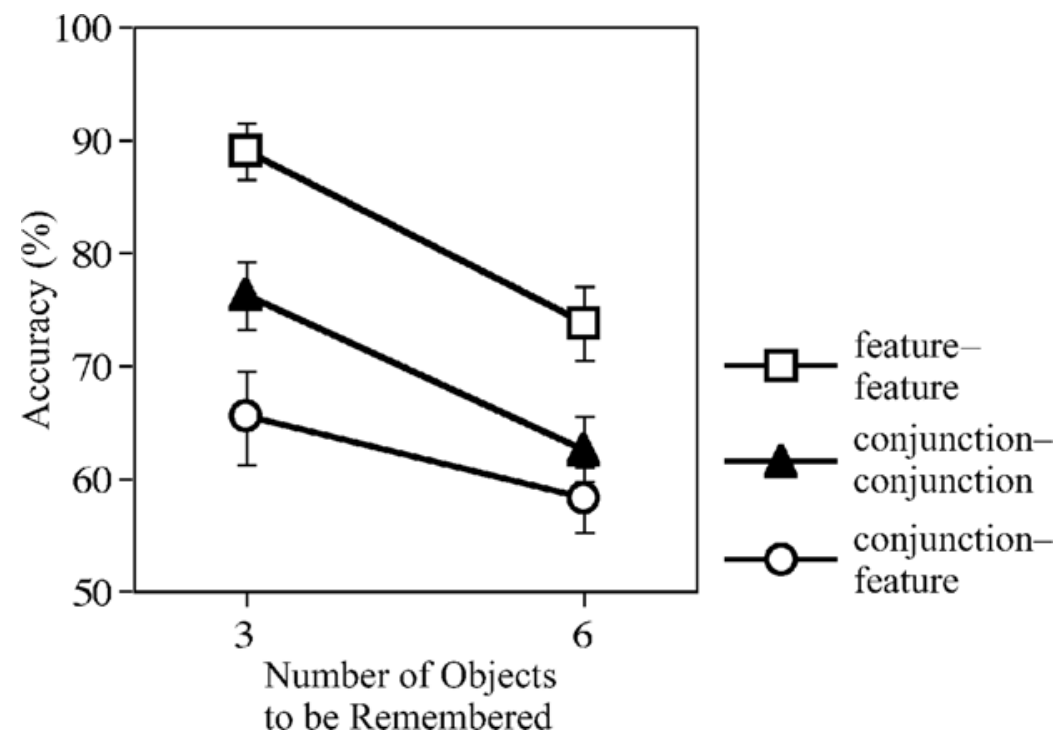

Figure 2. Results from Experiment 1, with mean accuracy as a function of condition and the number of memory objects. Error bars represent standard error of the mean.

One could argue that this failure to replicate the object benefit was due to strategic factors on the part of our observers. Perhaps mixing the feature and conjunction memory displays within a block encouraged our observers to adopt a feature, rather than an object, mode. Perhaps our observers simply chose to process individual features regardless of whether they formed a conjunction object. This criticism, however, is clearly invalid. When the memory display contained conjunction objects, VSTM was impaired if the probe item was a single feature rather than a conjunction object, indicating that the two colors in a conjunction object were encoded and processed in relation to each other. Thus, even when the observers tried to process the two colors of a conjunction object relationally, their memory was not improved by feature conjunction.

Thus, our failure to replicate Luck and Vogel's (1997) and Vogel et al.'s (2001) results compromise the strong claim that VSTM only has a capacity limit for the number of objects. Our results indicate that when the the number of objects is held constant, VSTM is affected by the number of features that can be stored.

The results of Experiment 1 are inconsistent with the strong-object hypothesis. However, there are a number of reasons why we cannot yet reject the strong-object hypothesis, discussed below.

Our task and stimuli were similar to those used in the two previous studies (Luck \& Vogel, 1997; Vogel et al., 2001). However, some procedural differences exist that may have led to the particular pattern of results reported in Experiment 1. First, our probe display contained only a single object, whereas the previously noted studies used more complex probe displays containing all items from the memory display. It is unlikely, however, that this ac- counts for the discrepancy in results. Single-item displays are often used in change detection tasks because the elimination of irrelevant comparisons makes the decision process easier (see Irwin \& Andrews, 1996, as an example). Direct comparisons of single-probe and full-probe displays have sometimes shown a small decrement in memory accuracy in the single-probe condition produced by the removal of contextual items (Jiang et al., 2000). This effect, however, should apply equally to the conjunction and the feature memory tasks.

Second, we tested the feature and conjunction conditions in a single interleaved block, whereas, in the studies by Luck and colleagues (Luck \& Vogel, 1997; Vogel et al., 2001), conditions were tested in separate blocks. It is unclear which trial structure is preferable. On the one hand, interleaved trials may have led some of the participants to use a feature-oriented strategy preferentially to an objectoriented strategy, although as we noted earlier, the observers in Experiment 1 did not rely exclusively on a featureoriented strategy. On the other hand, interleaved trials are better for ruling out any strategies that the participants may have used within a particular set of trials. To better approximate the design of the original Luck and Vogel (1997) study, we used a blocked design in all other experiments reported in this paper.

Third, we did not use the exact colors tested by the previously cited authors. They used four CIE colors that were difficult to reproduce with our equipment. We used eight RGB colors. It is unclear which set of colors is more representative of the basis of VSTM. Hence, it is important to test more than one set of colors in additional experiments.

Finally, whereas observers in Luck and Vogel's (1997) study carried out an articulatory suppression task concur- 
rently with the VSTM task, our observers performed a single VSTM task. It is possible, therefore, that our observers might have relied partly on verbal STM to encode the visual stimuli. A verbal strategy may favor the feature mode of processing, since it is easier to verbally label a simple color object than a color-color conjunction object. This may have masked some effect of color-color conjunction in visual STM.

To resolve the discrepancy between our study and Luck and Vogel's (1997) study, we conducted Experiment 2 using testing procedures more similar to those used by Luck and Vogel.

\section{EXPERIMENT 2 Color-Color Conjunction: Choice of Colors, Verbal Load, and Blocked Design}

Compared with Experiment 1, Experiment 2 involved the following procedural changes: (1) A full probe instead of a single probe was used to test memory. On half of the trials, the full-probe display was identical to the memory display; on the other half of the trials, the full-probe display had a color change in one item. A rectangular frame indicated which item was to be compared with the memory image. (2) Conditions were blocked rather than randomly interleaved. (3) The duration of the memory image was reduced from 400 to $100 \mathrm{msec}$, the same duration used by Luck and Vogel (1997). (4) The observers held a verbal memory load of four digits throughout the VSTM trial to remove any impact of verbal STM on memory of the colors. (5) Two sets of colors, differing in saturation, were tested to allow results from one set of color to be compared with another set. The observers rated the saturation of the colors at the end of the session. (6) Finally, we removed the conjunction-feature condition tested in Experiment 1. Presumably, the block design used in Experiment 2 would encourage a strategy of representing the two colors of a conjunction object relationally. So we thought it was unnecessary to further demonstrate relational encoding.

The experiment involved a $2 \times 2 \times 2$ design, with condition (single feature vs. color-color conjunction), saturation (high- vs. low-salient colors), and set size as the main factors of interest, producing four blocked conditions each with two set sizes, tested in random order on individual observers. If any of the unique procedures used in Experiment 1 were responsible for our failure to support the strong-object hypothesis, then we should expect an object benefit in the present experiment, more clearly so for the high-saturation colors. In contrast, if VSTM of colors is barely affected by how the colors are conjoined for most color stimuli, we should not find any benefit for VSTM of conjoined color features.

\section{Method}

Participants. Nine naive students and employees from Yale University participated in this experiment. Their ages ranged from 18 to
25 years. All had normal color vision and normal or corrected-tonormal visual acuity.

Materials and Procedure. For the verbal load task, a modified version of Vogel et al.'s (2001) verbal load task was used. The observers were instructed to remember four randomly selected white digits that were presented side by side near the fovea for $1,000 \mathrm{msec}$ prior to trial onset. They were instructed to subvocally rehearse the digits throughout the trial. After a delay of $1,000 \mathrm{msec}$, the change detection memory task commenced. After the observers made their change detection decisions, and the screen was cleared, the observers were required to type the digits into the computer.

For the memory task, VSTM of colors was tested in the change detection paradigm similar to the one used in Experiment 1, except where noted. Exposure duration of the memory stimulus was $100 \mathrm{msec}$. There were four blocked conditions. The first condition, the high-saturation feature-feature condition, tested memory for single-color objects, similar to those in Experiment 1. The second condition, the low-saturation feature-feature condition, tested memory for single-color objects, using a new set of colors, described below. The third condition, the high-saturation conjunctionconjunction condition, tested memory for color-color conjunctions, as described in Experiment 1. The fourth condition, the low-saturation conjunction-conjunction condition, tested memory for color-color conjunctions using a new set of colors. The probe display was identical to the memory display on half of the trial and was changed in the color of one item on the other trials. To make clear which item should be compared with the memory image, a white rectangular marker enclosed the probe.

The low-saturation colors were less distinctive variants of those used previously: black $(55,55,55)$, white $(180,200,180)$, red $(170,0,0)$, pink $(190,0,180)$, yellow $(170,170,0)$, green $(50,178,50)$, blue $(50,50,160)$, and cyan $(0,175,175)$. These colors were selected on the basis of a pilot rating task indicating the low saturation of these colors. They were also less discriminable from one another, since they were more clustered together in the color space. All conditions were presented in a blocked format, randomized across observers. Each observer was tested in 15 practice trials ( 5 per condition) and 80 experimental trials per block (i.e., 2 [probe change absent vs. present] $\times 2$ [inner square or outer frame as the possible change feature] $\times 20$ cases).

For the rating task, at the end of the experiment, the observers rated the vividness of the colors used in the experiment on a scale from 1 to $10(1=$ least vivid, $10=$ most vivid $)$. The instructions were as follows:

\begin{abstract}
Please rate the color, from 1 to 10 , as to how vivid or saturated the color looks to you. Please keep in mind that we are not asking you to judge "brightness" because some colors, like black, can never be bright. Rather we are asking you to judge how well the color stands out from the background.
\end{abstract}

Each color was presented singularly for $100 \mathrm{msec}$ at fixation. Responses were entered with the number keys.

\section{Results}

Assessment of strong-object hypothesis. Trials with incorrect verbal recall were excluded from the analysis on the VSTM task. A total of $6 \%$ of trials were excluded by this measure. Mean accuracy as a function of the condition, number of to-be-remembered objects (i.e., set size), and color category is plotted in Figure 3. A repeated measures ANOVA tested the effects of Condition (2), set size (2), and the effect of color category (2). The main effect of condition was significant $[F(1,8)=54.77, p<.0001]$, with better performance for single-color objects than for color-color-conjunction objects, as was the main effect 


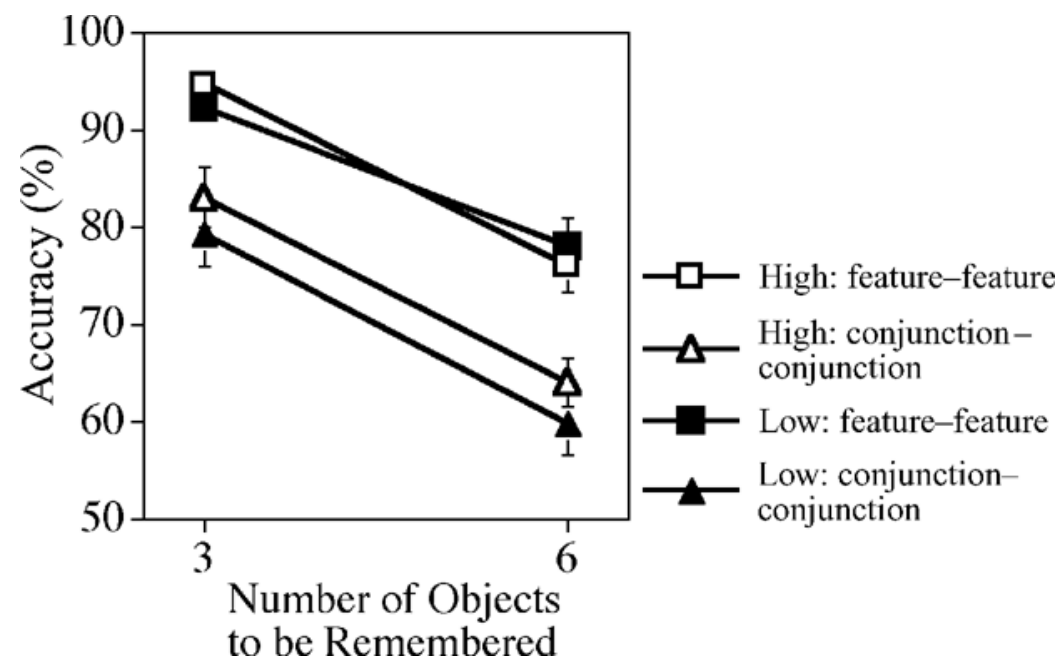

Figure 3. Results from Experiments 2, with mean accuracy as a function of condition, the number of memory objects, and color category. High refers to high-saturation colors. Low refers to low-saturation colors. Error bars represent standard error of the mean.

of set size $[F(1,8)=74.07, p<.0001]$, with better performance at the smaller set size. The main effect of color category approached significance $[F(1,8)=5.02, p<$ $.055]$, with better performance for high-saturation colors. None of the interaction effects were significant [condition $\times$ set size, $F(1,8)<1$; condition $\times$ color category, $F(1,8)=1.16, p>.30$; set size $\times$ color category, $F(1,8)<$ 1 ; and condition $\times$ set size $\times$ color category, $F(1,8)=$ $1.43, p>.25]$. Thus, holding the number of objects constant, VSTM was better for objects composed of one color than for objects composed of a conjunction of two colors, whether they were high-saturation or low-saturation colors. This is evidence against the strong-object hypothesis.

Assessment of the strong-feature hypothesis. Possible benefits of color-color conjunction in terms of the number of features remembered was determined as outlined in Experiment 1. We tested the difference between the six object (i.e., six features) feature-feature condition and the three-object (i.e., six features) conjunction conditions in a repeated measures ANOVA, with condition (2) and color saturation (2) as factors. The main effect of condition was not significant $[F(1,8)=3.36, p>.10]$, nor was the main effect of color saturation $(F<1)$ or their interaction $[F(1,8)=1.47, p>.20]$. Thus, the ANOVA test provided no evidence of conjunction benefit. Nevertheless, inspection of Figure 3 revealed that VSTM appeared to have benefited from conjunction in the high-saturation colors. Post hoc $t$ tests showed that, although conjunction had no effect on the low-saturation colors $[t(1,8)<1]$, it significantly enhanced VSTM for high-saturation colors $[t(1,8)=2.74, p<.025]$. Thus, color saturation appeared to affect the conjunction benefit. Although the lack of interaction between color saturation and condition weakens this conclusion, our data conformed to Vogel et al.'s (2001) observation that the particular set of colors used in the test may be critical for observing conjunction effects.

Verbal load task. Performance on the verbal load task was high, ranging from $93 \%$ to $97 \%$ across various cells of the factorial design, with the average accuracy being 94\%. An ANOVA showed that verbal STM performance was not affected by any factors manipulated in the STM task (all $p \mathrm{~s}>.15$ ). The high verbal STM performance and its independence of the VSTM task suggests that the observers had devoted their verbal STM to the load task rather than to the VSTM task. Table 1 shows the accuracy data for the verbal STM task, as well as accuracy for the VSTM task under correct and incorrect verbal STM performance separately.

Table 1

Results from Experiment 2 (in Percentages)

\begin{tabular}{|c|c|c|c|c|c|c|c|c|}
\hline & \multicolumn{8}{|c|}{ Condition } \\
\hline & \multicolumn{4}{|c|}{ Feature-Feature } & \multicolumn{4}{|c|}{ Conjunction-Conjunction } \\
\hline & \multicolumn{2}{|c|}{ High } & \multicolumn{2}{|c|}{ Low } & \multicolumn{2}{|c|}{ High } & \multicolumn{2}{|c|}{ Low } \\
\hline VSTM $\mid$ Correct & 95 & 77 & 93 & 78 & 84 & 65 & 80 & \\
\hline VSTM|Incorrect verbal STM & 92 & 62 & 89 & 79 & 80 & 54 & 76 & 58 \\
\hline Error rate for verbal STM & 3.3 & 5.8 & 5.0 & 5.3 & 6.9 & 7.2 & 5.8 & 6.7 \\
\hline
\end{tabular}

Note-High refers to the high saturation of colors. Low refers to the low saturation of colors. The numbers of objects for the high and low saturation of colors were three and six, respectively. VSTM, visual short-term memory. Verbal STM, verbal short-term memory. 
We examined the performance of VSTM as a function of the verbal STM accuracy. If the observers had used a verbal encoding strategy, the verbal load task and the VSTM task would be competing for the same verbal short-term store. This would predict a negative correlation between verbal STM and VSTM performance. Table 1 shows that this was not the case. VSTM was poorer in nearly all conditions when verbal STM was incorrect, suggesting a positive, rather than a negative, correlation. The participants may have had a lapse of attention on some trials that led to errors on both verbal STM and VSTM tasks. Thus, there was no evidence that the observers used a verbal encoding strategy in the VSTM task. Experiment 2, and, hence, our failure to support the strong-object hypothesis truly tapped the process of VSTM.

Color rating task. All subjects rated the saturated colors as more vivid $(M=7.76)$ and the unsaturated colors as less vivid $(M=4.66)$. This difference was significant $[t(8)=11.25, p<.0001]$.

\section{Discussion}

Just as in Experiment 1, Experiment 2 failed to support the strong-object hypothesis of VSTM representation. VSTM was clearly better in the simple-feature condition than in the color-color conjunction condition. In other words, the number of features per object matters in VSTM. Experiment 2 also showed that the specific colors used for testing VSTM in Experiment 1 were not unique; the additional set of colors used in Experiment 2 led to the same failure to find an object benefit. Holding the number of objects constant, the VSTM advantage for the simplefeature condition was the same, irrespective of whether high-saturation colors or low-saturation colors were to be memorized. Vogel et al. (2001) suggested that choice of highly discriminable colors was an important aspect of their experimental manipulation. The colors used in the saturated condition of Experiment 2 were also more discriminable. One could argue that even these colors were not saturated and distinctive enough to produce an object benefit. But this would indicate that the object-based effect was peculiar to specific sets of colors, limiting its utility as a general rule of VSTM representation. The strongobject hypothesis was rejected by testing on not one but two sets of colors that differ in vividness and distinctiveness.

Memory of features was not improved when they were grouped to form fewer numbers of objects. This was true particularly for the low-saturation colors. There was suggestive evidence that conjunction may play a minor role for high-saturation colors because VSTM for conjoined high-saturation colors was better than VSTM for single high-saturation colors. This result is consistent with the possibility that the different pattern of results found across laboratories may be due to the specific stimuli used. Other procedural factors, such as blocked versus interleaved conditions, presence or absence of verbal load, full versus single probe, or short $(100 \mathrm{msec})$ versus long $(400 \mathrm{msec})$ memory duration, cannot account for our failure to support the strong-object hypothesis. Thus, Experiment 2 narrowed down the possible critical difference between our study and Luck and Vogel's (1997) study to the specific colors of the stimuli. This point is expanded more in the General Discussion section.

Experiments 1 and 2 provide evidence against the idea that the visual system is always able to use any kind of object conjunction to enhance STM, rejecting the strongobject theory proposed by Luck and Vogel (1997). However, it is premature to conclude that VSTM in no way uses object-based representations as suggested by the strong-feature hypothesis. Color-color conjunctions may be an unusual type of conjunction object. Color-color conjunctions are formed when parts of objects are conjoined, whereas color-orientation conjunctions are formed when properties of an object are conjoined. Parts-based integration forms worse objects than property-based integration (Xu \& Potter, 1999). To more fully assess the objectbased theory of VSTM, we tested VSTM for objects with property-based integration. Experiments 3 and 4 were designed to find out which of the three hypotheses provides a preferable characterization of VSTM.

\section{EXPERIMENT 3 Size-Orientation Conjunctions in VSTM}

In Experiment 3, we manipulated features from two different dimensions - size and orientation - to examine whether the number of features is all that matters in VSTM. This experiment also served to replicate Luck and Vogel's (1997) conjunction effect with different feature types integrated at the same location.

\section{Method}

Participants. Eight new observers were tested in this experiment. They were selected from the same subject pool as in Experiment 1.

Materials. Three types of stimuli were used: (1) white oriented lines, $1.0^{\circ} \times 0.1^{\circ}$, selected from four possible orientations $\left(0^{\circ}, 45^{\circ}\right.$, $90^{\circ}$, and $\left.135^{\circ}\right)$; (2) four sizes of horizontal white lines $\left(0.7^{\circ}, 1.4^{\circ}\right.$, $2.0^{\circ}$, and $2.5^{\circ}$ in length); and (3) a conjunction of orientation and size in the form of a variably sized white line. Orientations and sizes were the same as those used in the first two types of objects. The stimuli appeared within an invisible $8 \times 8$ cell matrix that subtended $17^{\circ} \times 13^{\circ}$.

Procedure. VSTM was tested in the same change detection paradigm as that used in Experiment 1. Each observer was tested in three separate blocked conditions. They were instructed to perform a change detection task in each block. Every trial was composed of a memory image (400 $\mathrm{msec})$, a delay period $(907 \mathrm{msec})$, and a single probe item (until response). The probe either was identical to the memory item at that position or changed one feature.

Three conditions were tested. In the orientation simple-feature block, all elements (two, four, or eight) on the display were the same size, but in different orientations. In the size simple-feature block, all elements (two, four, or eight) on the display were of the same orientation (horizontal), but in different sizes. In the conjunction block, each element was a conjunction of the two simple features: size and orientation. The number of elements on the display was either two or four, corresponding to four or eight features. The observers were instructed to hold the size or the orientation of the lines in memory in the simple-feature blocks and to hold both the size and the orientation in memory in the conjunction block.

The observers performed five practice trials. There were 48 trials in each of the feature blocks: $48=3$ (set size) $\times 2$ (change present 


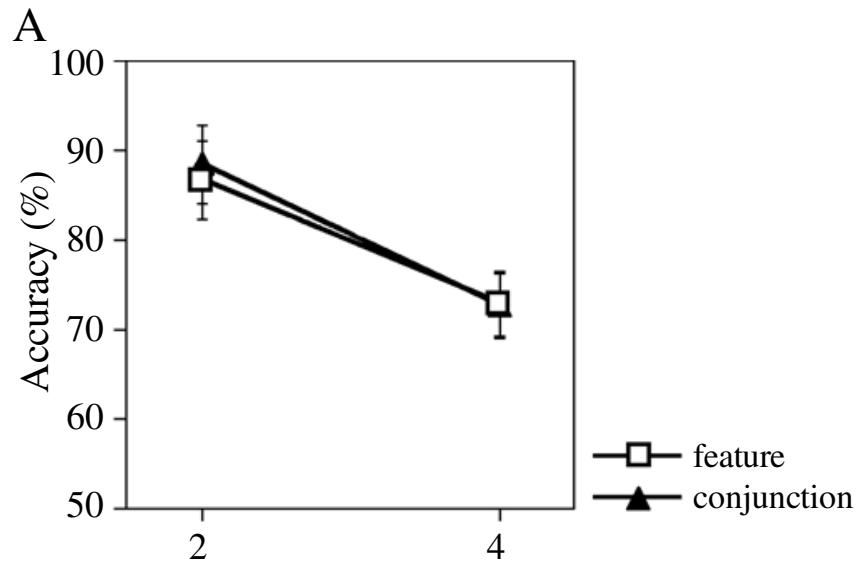

B

Number of Objects
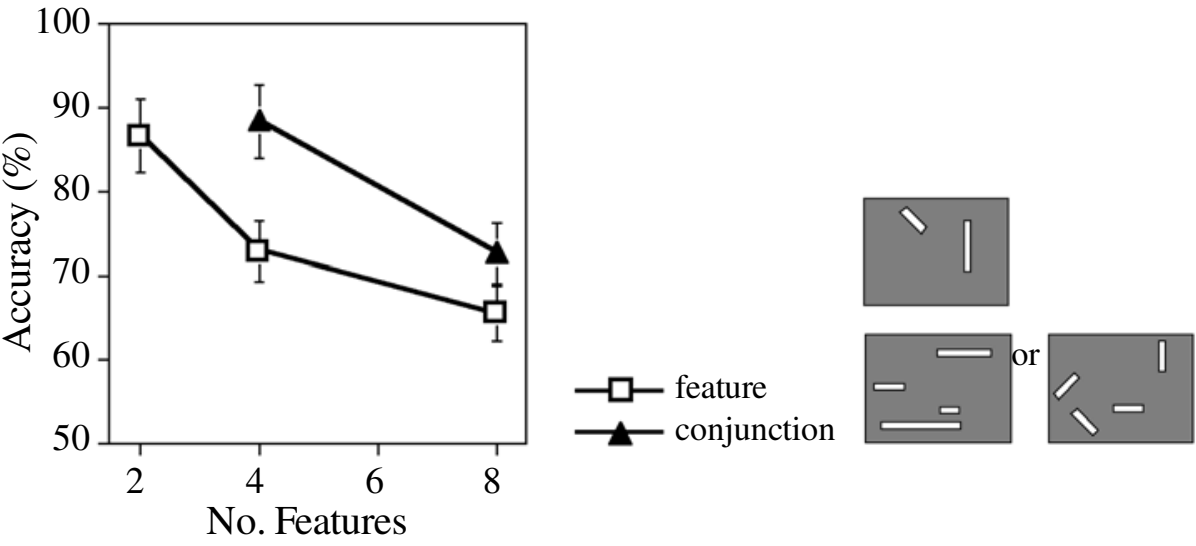

Figure 4. Results from Experiment 3, orientation-size conjunctions. (A) Mean accuracy as a function of condition and the number of memory objects. Sample memory displays were also shown on the right side of the figure. (B) Mean accuracy as a function of condition and the number of memory features. Error bars represent standard error of the mean.

or absent) $\times 2$ types of features (color squares or oriented lines) $\times$ 8 cases. A total of 96 trials were tested in the conjunction block: $96=2$ (set size $) \times 2$ (change present or absent) $\times 24$ cases. Block order was randomly assigned to each observer.

Other aspects of the experiment were the same as those in Experiment 1 .

\section{Results}

Assessment of strong-object hypothesis. Figure 4A plots mean accuracy as a function of the number of to-beremembered objects and conditions; Figure $4 \mathrm{~B}$ plots mean accuracy as a function of the number of to-be-remembered features and conditions. The number of to-be-remembered objects was equal to the number of features in the two feature conditions and was half the number of features in the conjunction condition. Samples of the memory displays of the three blocks are also illustrated in Figure 4B.

We first tested VSTM performance between the simplefeature conditions. Because accuracy did not differ between the length and orientation conditions $(F<1)$, we collapsed the data from these two conditions into one simple-feature condition. Accuracy in this condition was compared with that in the conjunction condition when the number of objects (i.e., set size) was two and four (see Figure 4A). The main effect of the number of objects was significant $[F(1,7)=87.84, p<.001]$; however, in sharp contrast to Experiments 1 and 2, the main effect of condition was not significant $(F<1)$, nor was the interaction between condition and set size $(F<1)$. Thus, performance was equivalent between these two conditions when assessed against the number of to-be-remembered objects, replicating Luck and Vogel's (1997) results and supporting the strong-object hypothesis.

Assessment of strong-feature hypothesis. We held the number of to-be-remembered features constant at four and eight and tested the difference between conditions (see Figure 4B). We found a significant main effect of the number of features $[F(1,7)=21.76, p<.002]$, with better performance at the smaller set size. There was a significant main effect of condition $[F(1,7)=9.26, p<$ $.019]$, with better performance when features were integrated, and there was a significant interaction $[F(1,7)=$ $6.29, p>.041]$, showing a more pronounced conjunction 
benefit at the smaller set size. Planned comparisons showed that accuracy was significantly higher in the conjunction condition than in the simple-feature condition when there were four features $[t(7)=6.49, p<.001]$ and eight features $[t(7)=2.94, p<.022]$. Thus, putting size and orientation in conjunction improved memory for features, rejecting the strong-feature hypothesis.

\section{Discussion}

Experiment 3 successfully replicated Luck and Vogel's (1997) results for integrated features from different feature domains. We found that, when the number of objects was held constant, memory performance was similar for orientation, size, and their conjunction. These results confirm an important conclusion from Luck and Vogel's study: VSTM capacity of features can be increased through feature conjunction.

Experiment 3 lent apparent support for the strongobject hypothesis (Luck \& Vogel, 1997). However, this hypothesis was clearly rejected in Experiments 1 and 2 . Likewise, the simplest version of the strong-feature hypothesis - that only the number of features matters-was clearly rejected in Experiment 3 . There are two theories left that can account for all of these results: the weak-object theory and the multiple-resources theory. The weak-object theory holds that VSTM capacity limits can be alleviated through the grouping of features into higher order chunks but that the efficiency of this process depends on how features are conjoined and on how different the features are from one another.

The multiple-resources theory is congenial to the strong-feature hypothesis, granting a role only to features, not to objects. This theory assumes that there are separate memory resources for each feature dimension. It explains why memory capacity seems to have doubled in the conjunction condition by positing that two pools - the orientation and the size pools-were recruited. It also explains why memory capacity was not affected by color-color conjunctions, since the color memory pool is limited to a fixed number of colors.

Experiment 4 was designed to test whether the object benefit observed in Experiment 3 was better explained by the multiple-resources theory or by the strong-object hypothesis. If both theories are rejected, it can be assumed that the weak-object theory is the best characterization of VSTM.

\section{EXPERIMENT 4 \\ Color-Orientation Conjunctions in VSTM: Effect of Heterogeneity in To-Be-Remembered Features}

In Experiment 4, we tested VSTM for color-orientation conjunctions. The participants were tested in three conditions. In the homogeneous simple-feature condition, VSTM for a particular simple feature (either color squares or line orientations) was tested. Only one pool of memory resources should be recruited for these stimuli. In the heterogeneous simple-feature condition, VSTM for simple features of a mixed set (half items were color squares; the other half were line orientations) was tested. Two pools of memory resources should be recruited, potentially doubling the number of features held in these pools. In the conjunction condition, VSTM for color-orientation conjunctions was tested. Again, two pools of memory resources should be recruited.

The homogeneous simple-feature condition and the conjunction condition were adopted from Luck and Vogel's (1997) study. We expected to replicate their findings in these conditions. That is, we expected that the number of features that could be memorized would double in the conjunctioncondition, relative to the homogeneous simplefeature condition. The heterogeneous simple-feature condition is a new and critical condition that had not been tested in previous studies. Feature heterogeneity is comparable between the conjunction condition and the heterogeneous simple-feature condition, since both recruit two pools of memory resources. The multiple-resources theory predicts that accuracy should be equivalent in the heterogeneous simple-feature condition and in the conjunction condition and that both should retain twice as many features as the homogeneous simple-feature condition. In contrast, the strong-object hypothesis predicts that feature memory capacity should be doubled in the conjunction condition, relative to the heterogeneous feature condition, which should not differ from the homogeneous feature condition.

\section{Method}

Participants. Eight new observers were tested.

Materials. Three types of stimuli were used in Experiment 4: (1) single-colored squares $\left(0.6^{\circ} \times 0.6^{\circ}\right)$ whose colors were selected from the eight possible colors used in Experiment 1; (2) white oriented lines $\left(1.0^{\circ} \times 0.1^{\circ}\right)$, selected from eight possible orientations $\left(0^{\circ}, 22.5^{\circ}, \ldots\right.$, and $157.5^{\circ}$, incremented by a step of $\left.22.5^{\circ}\right)$; and (3) conjunction of color and orientation in the form of colored lines-orientations and colors were the same as those used in the first two types of objects.

Procedure. VSTM was tested in the change detection paradigm. Each observer was tested in three separate blocked conditions. Every trial was composed of a memory image $(400 \mathrm{msec})$, a delay period (907 $\mathrm{msec})$, and a full-probe image (until response). The two images were identical except that, in the probe display, one element might change one feature on $50 \%$ of the trials. The critical item was indicated by an outline frame.

Three conditions were tested. In the homogeneous simple-feature block, all elements on the same display were of the same type: white oriented lines or color squares, tested in separate blocks. There were three set sizes in this block: two, four, or eight. The features on the same display could repeat at Set Size 8. In the heterogeneous simplefeature block, half of the items on the display were color squares, and the other half were white oriented lines at Set Size 4 or 8. In the conjunction block, each element was a conjunction of two simple features (i.e., color and orientation) at Set Size 2 or 4, corresponding to four or eight features. The observers were instructed to hold the color of the color squares or the orientation of the white lines in memory in the homogeneous simple-feature blocks and the heterogeneous simple-feature block and to hold both the color and the orientation of the color lines in memory in the conjunction block.

The observers performed five practice trials per block. They were tested on 48 trials in each of the two homogeneous simple-feature blocks and 96 trials in the heterogeneous simple-feature block or the 


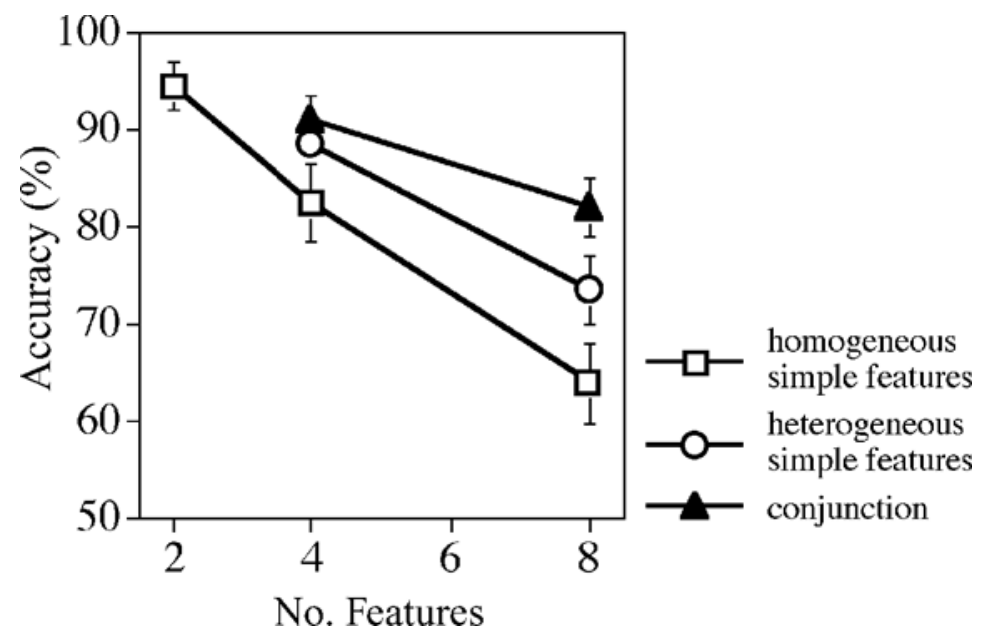

Figure 5. Results from Experiment 4, orientation-color conjunctions. Mean accuracy as a function of condition and the number of memory features. Error bars represent standard error of the mean.

conjunction block. Block order was randomly assigned to each observer.

Other aspects of the experiment were the same as those in Experiment 1 .

\section{Results}

Assessment of the strong-object hypothesis. Mean accuracy as a function of the number of to-be-remembered features and conditions is plotted in Figure 5. Note that, to make the figure more readable, we put the number of tobe-remembered features on the horizontal axis.

We first compared VSTM performance between the conjunction and the homogeneous feature conditions when the number of objects was two and four. The main effect of condition was not significant $[F(1,7)<1]$, since performance was equivalent between these two conditions when assessed against the number of to-be-remembered objects, replicating Luck and Vogel's (1997) results. However, multiple pools of memory resources might aid the conjunction condition. The more appropriate comparison should be drawn between the conjunction and heterogeneous feature conditions, because these were matched in the pools of hypothetical memory resources. These two conditions shared only one set size level when assessed against the number of objects (4). Memory performance was $80 \%$ in the conjunction condition when the object number was 4. This was significantly lower than performance in the heterogeneous feature condition at Set Size $4(M=89 \%)$ $[t(7)=2.68, p<.032]$. Thus, storing two features of an object produced an impairment, relative to storing one feature of an object, rejecting the strong-object hypothesis.

Assessment of the strong-feature hypothesis. The number of to-be-remembered features was held constant at four and eight, and the difference was tested between conditions. There was a significant main effect of the number of features $[F(1,7)=36.89, p<.001]$, with better performance at smaller set sizes, a significant main effect of condition $[F(2,14)=14.65, p<.001]$, and a nonsignificant interaction $[F(2,14)=1.79, p>.20]$. Planned comparisons showed that accuracy was significantly higher in the conjunction condition than in the homogeneous feature condition $[F(1,7)=18.61, p<.004]$ and was significantly higher than the heterogeneous feature condition $[F(1,7)=6.88, p<.034]$. The latter effect indicates that putting color and orientation in conjunction improved memory performance, even when the number of pools of memory resources was controlled for, rejecting the multiple-resources theory as a satisfactory explanation for all data reported in this study. Finally, the heterogeneous feature condition had significantly higher accuracy than did the homogeneous feature condition $[F(1,7)=12.45, p<.01]$, showing a heterogeneity effect.

\section{Discussion}

Experiment 4 tested whether the VSTM object benefit observed in Experiment 3, Luck and Vogel (1997), and Vogel et al. (2001) was better explained by the strong-object theory or by the multiple-resources theory of VSTM.

The results of Experiment 4 failed to support either theory. The strong-object theory was rejected, because when the number of objects was held constant, VSTM was better in the heterogeneous feature condition than in the conjunction condition, suggesting that it was more difficult to store two features than one feature of an object in VSTM.

The multiple-resources theory was also rejected, because when the number of features was held constant, VSTM was better when features conjoined to form objects than when they were isolated features. This was true even when heterogeneity was controlled for in the comparison.

On the basis of these findings, we conclude that the observed object benefit is best explained by the weak-object hypothesis that granted a role for both the number of objects and the number of features in VSTM. 


\section{GENERAL DISCUSSION}

In the introduction to this paper, we suggested that there are three competing hypotheses about VSTM capacity limitations: the strong-objecthypothesis, the strong-feature hypothesis, and the weak-object hypothesis. Experiments 1 and 2 used conjunction objects defined by colors only. Conjunctions were formed by the juxtaposition of different parts. In Experiment 1, we found that the participants had more accurate memories for single-colored objects than for bicolored objects. In Experiment 2, we found similar results generalized to two sets of colors differing in saturation. For these stimuli, the number of features played a dominant role in limiting the capacity of VSTM.

Although Experiments 1 and 2 provide evidence against the strong-object hypothesis, they are not representative of all kinds of conjunctions. The merit of using colorcolor conjunction is that the results would provide evidence for or against a multiple-resources theory in which separate memory capacities exist for different feature dimensions. The drawback is that color-color juxtaposition by no means exhausts different kinds of feature conjunctions.

To test whether the findings from Experiments 1 and 2 generalized to conjunction objects formed by feature integration, in Experiment 3, we tested conjunction objects defined by size and orientation, allowing conjunction to be formed by integrating different properties at the same location. The results showed that, when the number of objects was held constant, conjunction objects were remembered as well as single-feature objects, replicating Luck and Vogel's (1997) finding.

These results show that VSTM is enhanced when features conjoin to form objects. Because Experiments 1 and 2 clearly rejected the strong-object hypothesis and because Experiment 3 rejected the strong-feature hypothesis, two theories remained that could account for all of these results: the weak-object theory and the multiple-resources theory. The multiple-resources theory was tested against the strong-object hypothesis in Experiment 4, in which we compared feature and conjunction of color-orientation after controlling for feature heterogeneity. Results showed that, when the number of features was held constant, VSTM of color-orientation conjunction was better than, but not twice as good as, VSTM of each individual feature. Thus, both features and objects play a role in VSTM, supporting the weak-object hypothesis.

The results of Experiment 4 suggest that the heterogeneity of the to-be-remembered features is an important factor affecting performance in VSTM. VSTM is better when features are more heterogeneous. Previous VSTM studies failed to control for heterogeneity, and it is likely that the enhanced VSTM in the conjunction conditions was a joint effect of conjunction benefit and feature heterogeneity, leading to problems in interpretation. In Experiment 4 , we isolated these two effects by controlling for feature heterogeneity. We note, however, that the origin of the heterogeneity effect may not stem from VSTM but rather from visual attention. At least part of the VSTM benefit of representing heterogeneous features arises from an enhanced efficiency of attention to these features (Duncan \& Humphreys, 1989). Regardless of its exact origin, its existence justifies a necessity to control for feature heterogeneity when comparing VSTM of features with conjunctions.

Why did we fail to replicate the object-based effect for VSTM of color-color conjunctions? Results from Experiment 2 hint that the specific stimulus colors can influence whether memory is more or less object based. It is possible that the colors used by Luck and Vogel (1997) were special, being highly distinctive and saturated. Why might specific colors be so important? One possibility is that unusually vivid, high-contrast colors allow observers to memorize salient color contrast cues of a color-color conjunction object rather than individual colors. Instead of trying to remember each of the two colors in a conjunction object, observers might have simply encoded the contrast between the two colors. Such a strategy would reduce the number of features from two (colors) to one (contrast). Experiment 1 provided suggestive evidence that the depletion of high color contrast during retrieval produced memory impairment. However, the contrast cue appeared not strong enough to produce any object benefit in Experiment 1, although it produced a noticeable object effect in Experiment 2. Lowering the saturation of the colors eliminated the object benefit completely in Experiment 2. In this interpretation, the object benefit obtained with high-saturation colors is not valid evidence for the strong-object hypothesis.

To conclude, we have shown in this study that the weakobject theory may characterize the representation of VSTM the best. But we caution that the success of this theory partly lies in its generous conjecture of the roles of features and objects giving room for additional assumptions to be added to account for a particular set of data. The validity of this theory requires a more stringent characterization of several factors that influence VSTM, such as how features are conjoined into an object and why objectbased or feature-based effects occur when they do occur. The weak-object hypothesis is not complete without future delineation of these important factors.

\section{REFERENCES}

Baddeley, A. (1986). Working memory. Oxford: Oxford University Press.

Bower, G. H., \& Winzenz, D. (1969). Group structure, coding, and memory for digit series. Journal of Experimental Psychology, 80 (2, Pt. 2), 17.

Cowan, N. (2001). The magical number 4 in short-term memory: A reconsideration of mental storage capacity. Behavioral \& Brain Sciences, 24, 87-185.

DunCAN, J. (1984). Selective attention and the organization of visual information. Journal of Experimental Psychology: General, 113, 501517.

Duncan, J., \& Humphreys, G. W. (1989). Visual search and stimulus similarity. Psychological Review, 96, 433-458.

Egly, R., Driver, J., \& Rafal, R. D. (1994). Shifting visual attention between objects and locations: Evidence from normal and parietal le- 
sion subjects. Journal of Experimental Psychology: General, 123, 161-177.

Ericsson, K. A., Chase, W. G., \& Faloon, S. (1980). Acquisition of a memory skill. Science, 208, 1181-1182.

Hunt, S. M. J. (1994). MacProbe: A Macintosh-based experimenter's workstation for the cognitive sciences. Behavior Research Methods, Instruments, \& Computers, 26, 345-351.

InTRAub, H. (1997). The representation of visual scenes. Trends in Cognitive Sciences, 1, 217-222.

IRwIN, D. E., \& ANDREws, R. V. (1996). Integration and accumulation of information across saccadic eye movements. In T. Inui \& J. L. McClelland (Eds.), Attention and performance XVI: Information integration in perception and communication (pp. 125-155). Cambridge, MA: MIT Press.

JiAng, Y., Olson, I. R., \& Chun, M. M. (2000). Organization of visual short-term memory. Journal of Experimental Psychology: Learning, Memory, \& Cognition, 26, 683-702.

Lee, D., \& Chun, M. M. (2001). What are the units of visual short-term memory, objects or spatial locations? Perception \& Psychophysics, 63, 253-257.

LogIE, R. H. (1995). Visual-spatial working memory. Hillsdale, NJ: Erlbaum.

Luck, S. J., \& VogeL, E. K. (1997). The capacity of visual working memory for features and conjunctions. Nature, 309, 279-281.

Macmillan,N. A., \& Creelman, C. D. (1991).Detection theory: A user's guide. New York: Cambridge University Press.

Miller, G. A. (1956). The magical number seven, plus or minus two: Some limits on our capacity for processing information. Psychological Review, 63, 81-97.

PAshler, H. (1988). Familiarity and visual change detection. Perception \& Psychophysics, 44, 369-378.

Phillips, W. A. (1974). On the distinction between sensory storage and short-term visual memory. Perception \& Psychophysics, 16, 283-290.
Simon, H. A. (1974). How big is a chunk? Science, 183, 482-488.

Simons, D. J. (1996). In sight, out of mind: When object representations fail. Psychological Science, 7, 301-305.

Sperling, G. (1960). The information available in brief visual presentations. Psychological Monographs: General \& Applied, 74, 1-19.

Tulving, E., \& Thomson, D. M. (1973). Encoding specificity and retrieval processes in episodic memory. Psychological Review, 80, 352373.

Vecera, S. P., \& Farah, M. J. (1994). Does visual attention select objects or locations? Journal of Experimental Psychology: General, 123, 146-160.

Vogel, E. K., Woodman, G. F., \& Luck, S. J. (2001). Storage of features, conjunctions, and objects in visual working memory. Journal of Experimental Psychology: Human Perception \& Performance, 27, 92114.

Wheeler, M. E., \& Treisman, A. M. (1999).Aspects of time, space and binding in visual working memory for simple objects. Poster presented at the 7th Annual Workshop on Object Perception and Memory, Los Angles.

Wolfe, J. M., Yu, K. P., Stewart, M. I., Shorter, A. D., FriedmanHill, S. R. \& CAVE, K. R. (1990). Limitations on the parallel guidance of visual search: Color $\times$ color and orientation $\times$ orientation conjunctions. Journal of Experimental Psychology: Human Perception \& Performance, 16, 879-892.

Xu, Y., \& Potter, M. C. (1999). Objects through the eyes of visual working memory. Poster presented at the 7th Annual Workshop on Object Perception and Memory, Los Angeles.

(Manuscript received May 7, 2001; revision accepted for publication December 27, 2001.) 